\title{
Identification of a high-affinity RNA-binding site for the human immunodeficiency virus type 1 Rev protein
}

\author{
Laurence S. Tiley*, Michael H. Malim*†, Hamant K. Tewary ${ }^{*}$, Peter G. Stockley $\ddagger$, \\ AND BRYAN R. CULlEN ${ }^{*}+\S \uparrow$ \\ ${ }^{*}$ Howard Hughes Medical Institute, ${ }^{\S}$ Section of Genetics, and ${ }^{\dagger}$ Department of Medicine, Duke University Medical Center, Durham, NC 27710 ; and
${ }^{\ddagger}$ Department of Genetics, University of Leeds, Leeds, LS2 9JT, United Kingdom
}

Communicated by Joan A. Steitz, October 28, 1991 (received for review August 27, 1991)

\begin{abstract}
Expression of the structural proteins of human immunodeficiency virus type 1 requires the direct interaction of multiple copies of the viral Rev protein with its highly structured RNA target sequence, the Rev response element (RRE). Nucleotides critical for Rev monomer binding have been mapped by chemical interference to a single site flanking the base of an RNA helix (stem IIB) located within the 234-nucleotide RRE. Binding of additional Rev molecules to an RRE probe did not require any RNA primary sequence information detectable by modification interference beyond that required for binding of a single Rev protein molecule. A synthetic 29-nucleotide RNA molecule designed to incorporate nucleotides identified as critical for Rev binding retained the ability to bind Rev specifically and, therefore, represents a minimal Rev-binding site. We propose that Rev binding to the RRE initiates with the direct interaction of a Rev monomer with a high-afinity binding site located at the base of the IIB stem of the RRE. The subsequent formation of Rev multimers on the RRE appears, in contrast, primarily driven by specific protein-protein interactions.
\end{abstract}

The Rev gene product of human immunodeficiency virus type 1 (HIV-1) is an RNA-sequence-specific nuclear regulatory protein essential for HIV-1 replication in culture (1-6). The target sequence for Rev, the rev response element (RRE), was initially defined as a complex 234-nucleotide (nt) RNA stem-loop structure located within the viral env gene (7). At least two, and possibly as many as eight, Rev molecules have been shown to bind specifically to the full-length $R R E$ in vitro (3-5, 8-10). Footprinting experiments suggest that these Rev protein molecules occupy discrete sites on the RRE (10). Although we and others have argued that the formation of Rev multimers on the RRE reflects the sequential binding of multiple Rev monomers $(8,9)$, it has also been suggested that the formation of multimers may be a prerequisite for Rev binding (11).

Mutational analysis of the 116-amino acid Rev protein has shown that an arginine-rich stretch of amino acids is critical for sequence-specific binding to the RRE, whereas flanking sequences are important for the formation of Rev multimers on the RRE $(8,11,12)$. It has therefore been proposed that this Rev "multimerization" is mediated, at least in part, by protein-protein interactions $(8,9,11,12)$. Rev mutants that have lost the ability to form multimers on the RRE in vitro display a recessive negative phenotype in vivo $(8,11)$.

Mutational analysis of the RRE has defined a 66-nt subdomain, termed stem-loop II (SLII) (Fig. 1A), that is both necessary and sufficient for high-affinity Rev binding in vitro $(4,5)$ and that displays at least partial RRE function in vivo (16). This RRE subdomain can bind up to three Rev molecules in vitro $(9,10)$. More recently, a 40-nt sequence

The publication costs of this article were defrayed in part by page charge payment. This article must therefore be hereby marked "advertisement" in accordance with $18 \mathrm{U}$ U.S.C. $\$ 1734$ solely to indicate this fact. contained within SLII has been proposed as a minimal RNA substrate based on its ability to bind a single Rev monomer (9). Here, we identify several noncontiguous nucleotides located within SLII that mediate Rev binding to the RRE. A 29-nt RNA oligomer that contains the minimal Rev-binding site predicted by this analysis retains the ability to bind $R e v$ specifically.

\section{MATERIALS AND METHODS}

Preparation of RNA Substrates. We have described (4) a plasmid, based on the in vitro transcription vector pGEM3Zf(+) (Promega) that contains the complete RRE SLII sequence. Unlabeled SLII RNA was synthesized by using SP6 RNA polymerase and purified as described (4). Oligoribonucleotides were synthesized by the solid-phase method on an Applied Biosystems model 391 DNA synthesizer using 2'-silyl-5'-dimethoxy-trityl phosphoroamidites (ChemGenes, Needham, MA). Synthesis and deprotection protocols were done essentially as described (17-19). Denaturing PAGE was used to purify full-length material. RNAs were $5^{\prime}$ end-labeled by using $\left[\gamma-{ }^{32} \mathrm{P}\right] \mathrm{ATP}(3000 \mathrm{Ci} / \mathrm{mmol}$, Amersham; $1 \mathrm{Ci}=37$ $\mathrm{GBq}$ ) and $\mathrm{T} 4$ polynucleotide kinase (New England Biolabs) or $3^{\prime}$ end-labeled by using [ $\left.5^{\prime}-{ }^{32} \mathrm{P}\right] \mathrm{pCP}(3000 \mathrm{Ci} / \mathrm{mmol}$, New England Nuclear) and T4 RNA ligase (New England Biolabs). End-labeled RNAs were subsequently repurified by electrophoresis through denaturing polyacrylamide gels.

RNA-Binding Assays. The recombinant 116-amino acid form of the HIV-1 Rev protein purified from Escherichia coli has been described $(3,4,8)$. Wild-type and mutant (M4) forms of Rev were also expressed in $E$. coli as GST fusion proteins and were purified as described (8). The conditions for in vitro complex formation between HIV-1 Rev and its target RNA and their visualization by native gel electrophoresis have been described $(4,8)$. A standard binding reaction contained $10 \mathrm{mM}$ Hepes (pH 7.6), 0.5 mM EGTA, 2 mM $\mathrm{MgCl}_{2}, 10 \%$ glycerol, $25 \mathrm{mM} \mathrm{NaCl}, 150 \mathrm{mM} \mathrm{KCl}, 1 \mathrm{mM}$ dithiothreitol, bovine serum albumin at $7.5 \mu \mathrm{g} / \mathrm{ml}$, and RNA guard at 100 units per $\mathrm{ml}$ (Pharmacia LKB). Each incubation (10 $\mu$ l) also included 500 ng of yeast tRNA as a nonspecific competitor, except where additional specific or nonspecific competitor was added, as indicated in the text.

Chemical Interference Assays. End-labeled purified SLII or SLIIB RNA samples were modified under denaturing conditions by using DEPC (15). Approximately $5 \times 10^{5} \mathrm{cpm}(0.5$ pmol) of chemically modified RNA was incubated with sufficient Rev protein to bind $\approx 25 \%$ of the RNA probe into higher-molecular-weight complexes. These complexes were resolved by using nondenaturing PAGE and visualized by autoradiography $(4,8)$. The "bound" and "free" RNAs were

Abbreviations: HIV-1, human immunodeficiency virus type 1; RRE, Rev response element; DEPC, diethylpyrocarbonate; SLII, stemloop II; nt, nucleotide(s); GST, glutathione $S$-transferase.

ITo whom reprint requests should be addressed. 

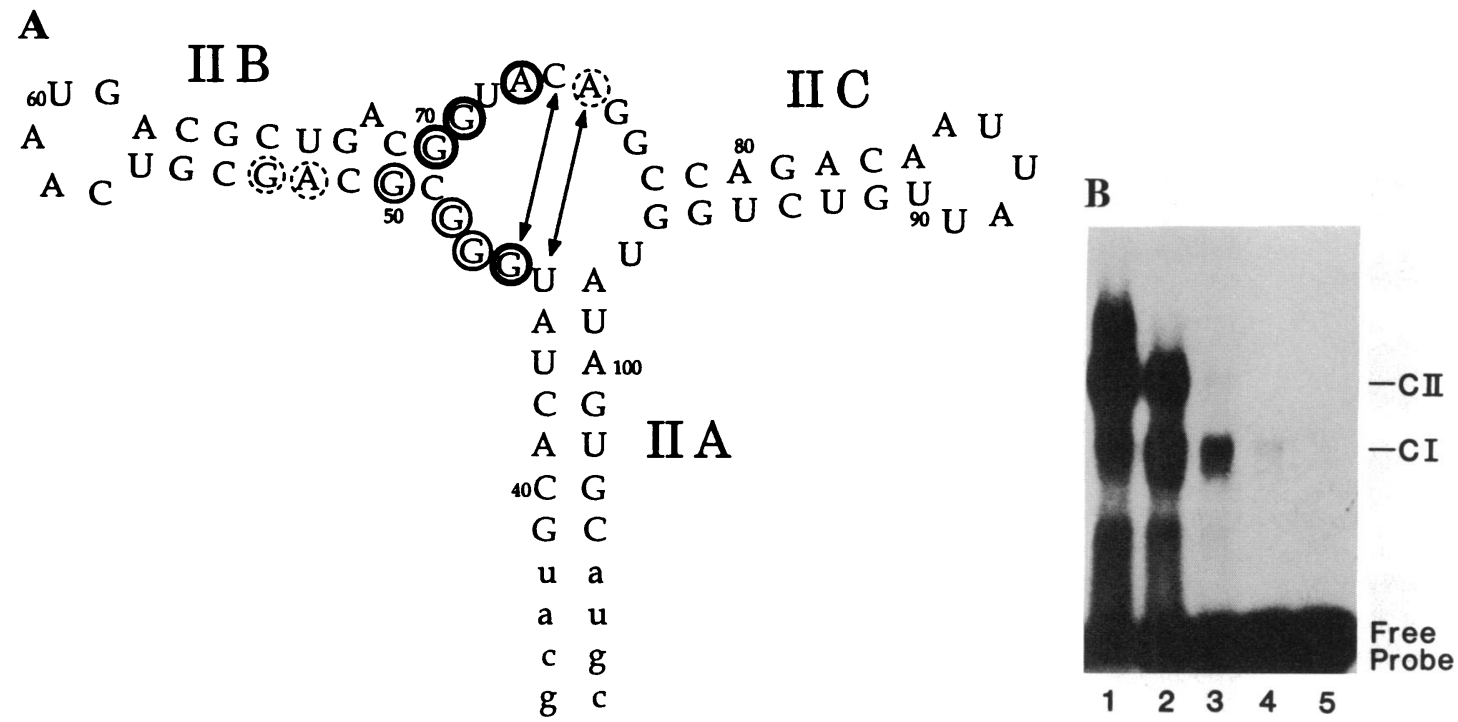

FIG. 1. The RRE SLII domain contains a high-affinity Rev binding site. (A) Computer-predicted secondary structure of the SLII domain (7). Nucleotides and RNA stem structures are numbered according to Malim et al. (7). The general validity of this secondary structure prediction has been confirmed both by mutational analysis and by analysis of in vitro nuclease sensitivity $(4,6,10,13,14)$. Arrows are used to indicate two additional base pairs predicted by the analysis of Dayton et al. (13). Individual nucleotides shown by chemical interference analysis as important for Rev binding are indicated: dark circles indicate strong interference $(>80 \%)$, light circles indicate substantial interference (50-80\%), and interrupted circles indicate mild interference (20-50\%), as determined in Fig. 2. Lowercase letters indicate a 4-nt extension of the IIA stem introduced during cloning (8). (B) Analysis of the interaction of the SLII probe with Rev. A constant level of a 5' end-labeled SLII RNA probe $\left(\approx 10^{4} \mathrm{cpm}\right.$ per lane), which had been chemically modified by diethylpyrocarbonate (DEPC) treatment (15), was titrated with increased levels of recombinant glutathione $S$-transferase (GST)-Rev protein (8). Binding of the probe was visualized as slower migration upon electrophoresis through a native polyacrylamide gel. As described $(4,8)$, at least two distinct specific protein-RNA complexes, termed CI and CII, are detected. Lanes: 1, $625 \mathrm{ng}$ of GST-Rev protein; 2, $125 \mathrm{ng}$ of GST-Rev; 3, $25 \mathrm{ng}$ of GST-Rev; 4, $5 \mathrm{ng}$ of GST-Rev; 5, no added protein.

excised, electroeluted with a Hoeffer GE 200 gel elutor, phenol-extracted, and ethanol-precipitated. The purified RNA samples were then subjected to aniline scission followed by three rounds of lyophilization (15). The cleaved RNA was analyzed by electrophoresis through denaturing $12 \%$ or $20 \%$ polyacrylamide gels. An alkaline hydrolysis ladder, as well as T1 and U2 RNase cleavage ladders generated from the unmodified test RNA, were run alongside the chemically cleaved RNAs to permit identification of individual bands after autoradiography.

\section{RESULTS}

To identify specific nucleotides within RRE SLII that are required for the interaction of Rev with the RRE, we examined the effect of limited chemical modification of bases within the SLII RNA probe on its ability to bind Rev. The modifying agent chosen, DEPC, carboxyethylates the $\mathbf{N}^{7}$ position of adenosine and, to a lesser extent, guanosine residues (15); this modification results in the opening of the purine imidazole ring and can interfere with protein-RNA interactions dependent upon the integrity of that base. A 5' end-labeled SLII RNA probe modified with DEPC was incubated with sufficient recombinant, 116-amino acid Rev protein to bind $\approx 30 \%$ of the input probe. The bound SLII RNA probe was then separated from free probe by nondenaturing PAGE. Subsequently, the free and bound RNA probe was recovered from the gel and treated with aniline, a reagent that cleaves the RNA adjacent to purine residues modified by DEPC (15), before electrophoresis through a $12 \%$ acrylamide/urea sequencing gel (Fig. 2). Purine residues, the modification of which interferes with the formation of Rev-RRE complexes, are identified by the absence of nucleotide-specific bands in the samples derived from the bound RNA (lanes 1) that are present in the free probe (lane 2). Inspection of Fig. 2 revealed several purine residues that play a role in Rev binding by the RRE. These purine residues, indicated in Fig. $1 A$, can be roughly divided into three classes by whether their modification by DEPC leads to complete interference $\left(G^{46}, G^{70}, G^{71}, A^{73}\right)$, substantial interference $\left(G^{47}, G^{48}, G^{50}\right)$, or mild interference $\left(A^{52}, G^{53}, A^{75}\right)$.

As noted above, the HIV-1 Rev protein can multimerize on the viral RRE to form a series of distinct protein-RNA complexes $(3-5,8-10)$. These complexes, which contain increasing numbers of Rev molecules bound to a single RRE $(8,9)$, can be resolved by nondenaturing gel electrophoresis. We therefore asked whether additional RNA sequence information is required for the binding of a second Rev molecule to an SLII RNA probe that is dispensable for the binding of a single Rev monomer. To identify the optimal ratio of Rev protein to SLII RNA for the formation of mono- and dimeric complexes, a constant level of the DEPC-modified, 5' endlabeled SLII probe was incubated with increased amounts of recombinant GST-Rev fusion protein (Fig. $1 B$ ). This fusion protein binds the RRE with high specificity and affinity, and, by virtue of its increased molecular weight, greatly enhances the resolution of Rev-RRE complexes (8). In agreement with previous reports (8-10), this titration (Fig. 1B) demonstrated the sequential appearance of complexes $C I$ and $C I I$ as the relative level of the GST-Rev protein was increased. The CI complex has been shown to contain a single Rev monomer bound to a single SLII RNA molecule, whereas CII contains two Rev monomers bound to the SLII RNA $(8,9)$.

Preparative gel-retardation analysis, under the conditions visualized in Fig. $1 B$, lane 2, was used to isolate SLII RNA molecules present in the $\mathrm{CI}$ and $\mathrm{CII}$ complexes, as well as free SLII RNA. As predicted, the GST-Rev fusion protein gave rise to a pattern of modification interference indistinguishable from the pattern observed with the 116-amino acid Rev protein (Fig. 2, lanes 1 and 4). More importantly, comparison of the interference pattern seen in the CI and CII Rev-RRE complexes with either $5^{\prime}$ end-labeled (Fig. 2, lane 4 vs. lane 5 ) or $3^{\prime}$ end-labeled (data not shown) probes revealed no detectable additional sequence requirement for formation of the CII complex beyond that seen for the CI complex. 


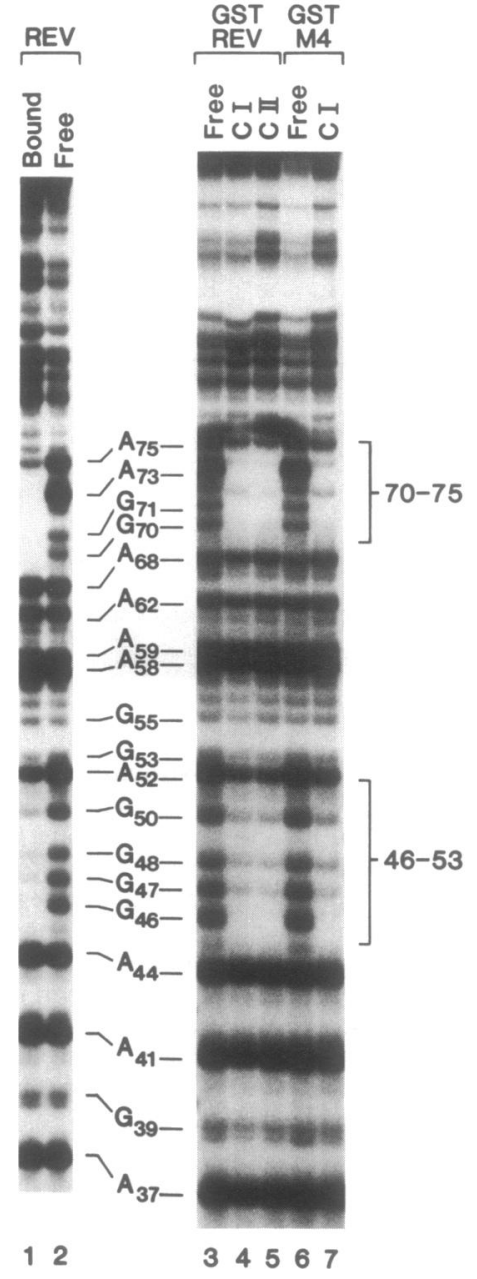

FIG. 2. Chemical interference analysis of Rev binding to the SLII RRE probes. A DEPC-modified, 5 ' end-labeled SLII RRE probe was incubated with sufficient 116-amino acid Rev protein (lane 1), GST-Rev protein (lanes 4 and 5), or GST-M4 protein (lane 7) to bind between $20 \%$ and $30 \%$ of the input probe. Free probe (lanes 2, 3, and 6) and either total bound probe (lane 1) or probe present in the $\mathrm{CI}$ (lanes 4 and 7) or CII (lane 5) protein-RNA complexes were isolated by native gel electrophoresis (Fig. $1 B$ ) and cleaved by treatment with aniline. A total of $4 \times 10^{3} \mathrm{cpm}$ derived from each RNA sample was subjected to electrophoresis through a denaturing $12 \%$ polyacrylamide gel, and the cleavage products were visualized by autoradiography. Nucleotides that displayed chemical interference are compiled in Fig. $1 A$.

We have described (8) a phenotypically negative mutant of Rev, termed M4, that binds the RRE with essentially normal affinity to form the monomeric CI complex but is then incapable of multimerizing on the RRE-i.e., no CII complex is formed. To address the RNA sequence specificity of this Rev mutant, we analyzed complex formation by M4 mutant on the modified SLII RNA probe in parallel with the wildtype Rev protein. The pattern of interference in the single CI complex formed by M4 mutant (Fig. 2, lane 7) was indistinguishable from that seen with wild-type Rev.

The data of Fig. 2 suggested that the RRE residues directly involved in binding Rev are clustered at the base of the IIB stem in SLII. This hypothesis predicts that a short RNA oligonucleotide that retained the IIB stem and flanking sequences might also retain the ability to bind Rev. Conversely, these chemical modifications could also have resulted in changes in RNA structure that indirectly interfered with RNA binding and, therefore, did not, in fact, map a binding site for Rev. In an attempt to distinguish between these two possibilities, we chemically synthesized (17-19) a 29-nt RNA, extending from residue 45 to residue 75 within the RRE but lacking residues 56 and 62 (Fig. $3 A$ ) and tested the ability of this SLIIB sequence to bind Rev specifically. Fig. $3 B$ shows that this 29-mer retained the ability to bind both the wild-type GST-Rev protein as well as the GST-M4 protein. However, the SLIIB probe differed from SLII and from the full-length RRE in that only a single protein-RNA complex was detected, even at very high levels of input GST-Rev protein. This protein-RNA interaction was specific, as it could be inhibited by unlabeled specific competitor (either SLIIB or SLII RNA) but not by similar levels of a nonspecific competitor, yeast tRNA (Fig. 3C). The observation that SLII RNA competed significantly more effectively than SLIIB RNA in this assay suggests that Rev has a higher affinity for SLII than for the minimal SLIIB oligonucleotide (see below).

To more completely determine the degree to which this SLIIB probe reproduced the interaction of $\operatorname{Rev}$ with the complete RRE SLII RNA, we performed a modification interference analysis on this RNA oligomer after end-labeling at either the 5 ' (Fig. 4, lanes 1 and 2) or $3^{\prime}$ (lanes 3 and 4) end. The results of this analysis, summarized in Fig. $3 A$, revealed a pattern of interference similar to that seen with the fulllength SLII sequence. Minor differences noted include a significant level of interference at residue $\mathrm{G}^{67}$ and an apparent enhancement in Rev binding upon modification of residue $A^{68}$.

\section{DISCUSSION}

The interaction of Rev with its cis-acting RNA response element, the RRE, is essential for the activation of HIV-1 structural gene expression and, hence, for viral replication (1-6). Although the full-length 234-nt RRE element is required for maximal RRE function in vivo, it has also been demonstrated that a 66-nt subdomain of the RRE, termed SLII, is both necessary and sufficient for high-affinity binding to $\operatorname{Rev}$ in vitro $(4,5)$. In this report, we have attempted to define specific nucleotides within SLII required for Rev binding. These experiments identified 10 purine residues clustered at the base of the SLIIB helix that, when modified, interfered with Rev binding (Fig. 1A). The hypothesis that these residues map the primary binding site of Rev on the RRE is supported by previous mutational analyses of the RRE $(4,6,13,14)$. In particular, the SLIIB stem has been shown to be required for Rev binding and RRE function, whereas the SLIIC stem is essentially dispensable $(4,6,9)$. Also the structural integrity of the IIB stem has been shown to be critical, rather than its specific sequence $(4,6)$. This result is consistent with our observation that Rev sequence specificity lies primarily at the base of the SLIIB stem and in the immediately flanking RNA sequence, rather than in the central helical portion of the stem. Our identification of RRE residues $G^{46}-G^{48}$ as important for Rev binding (Fig. $1 A$ ) is also consistent with a previous report that substitution of these three guanines with pyrimidine residues disrupts RRE function (14). The importance of these purine residues in RRE function is further supported by their conservation among cloned HIV-1 proviruses, including the highly divergent Zairian isolates (20).

Our analysis of Rev sequence specificity suggested that RRE stem IIB, together with its flanking sequences, represents a minimal Rev-binding site. This hypothesis was confirmed by the observation that a 29-nt RNA oligomer (Fig. $3 A$ ) that incorporated these sequences retained the ability to bind Rev specifically (Fig. $3 B$ and $C$ ) and displayed essentially the same chemical interference pattern as the complete SLII probe (Fig. 4). We note that this 29-nt SLIIB probe is completely contained within a 40-nt RRE sequence, extending from position 39 to 78 within SLII (Fig. 1A), which was recently shown by Cook et al. (9) to bind Rev. 

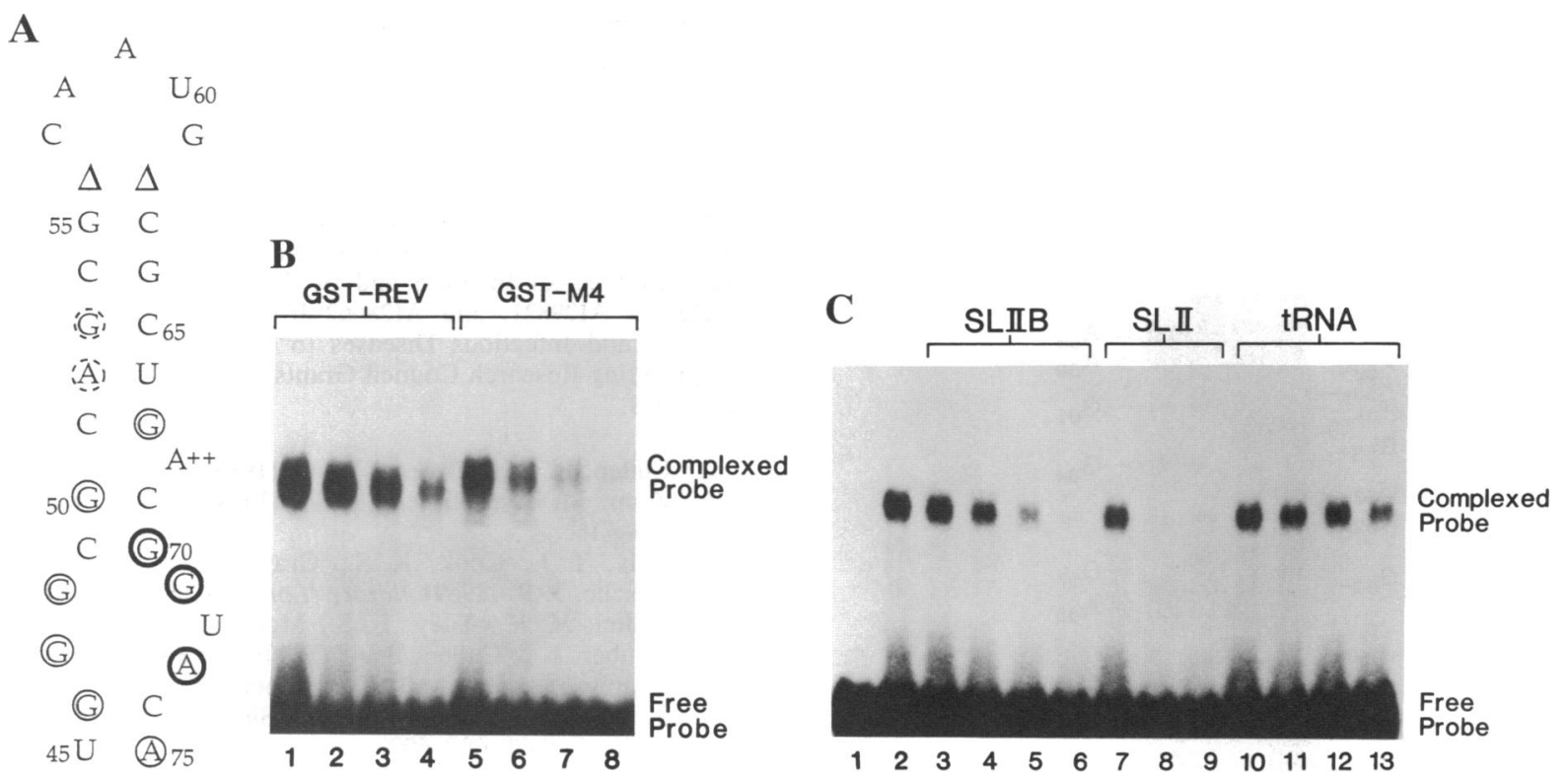

FIG. 3. The RRE SLIIB region represents a minimal Rev-binding site. (A) Computer-predicted (20-22) secondary structure of the 29-nt SLIIB probe. This sequence is derived from nt 45-75 of the RRE (Fig. $1 A$ ) but lacks $(\triangle)$ nt 56 (uridine) and nt 62 (adenosine). Individual nucleotides shown by chemical interference analysis (Fig. 4) to be important for Rev binding are indicated (see Fig. $1 A$ for code). The ++ adjacent to nt $A^{68}$ indicates that DEPC modification of this residue slightly enhances Rev binding (Fig. 4, lanes 3 and 4). (B) Analysis of the interaction of the indicated SLIIB probe with wild-type GST-Rev protein (lanes 1-4) or with GST-M4 Rev mutant (lanes 5-8). A constant level of ${ }^{32}$ P-end-labeled synthetic SLIIB probe was titrated with increased levels of the indicated Rev fusion proteins. Lanes 1 and $5,1 \mu \mathrm{g}$ of GST fusion protein; 2 and $6,333 \mathrm{ng}$ of protein; 3 and 7, $111 \mathrm{ng}$ of protein; 4 and 8, $37 \mathrm{ng}$ of protein. $(C)$ A constant level of the 3' end-labeled SLIIB RNA probe was incubated with $1 \mu \mathrm{g}$ of GST-Rev protein (except lane 1, which serves as a negative control) in the presence of increased levels of the indicated unlabeled competitor RNAs. Lanes: 1, SLIIB probe only; 2, no additional RNA competitor; 3, 10 ng of SLIIB RNA; 4, 50 ng of SLIIB RNA; 5, 250 ng of SLIIB RNA; 6, $1250 \mathrm{ng}$ of SLIIB RNA; 7, $10 \mathrm{ng}$ of SLII RNA; 8, $50 \mathrm{ng}$ of SLII RNA; 9, $250 \mathrm{ng}$ of SLII RNA; 10, $10 \mathrm{ng}$ of tRNA; 11,50 ng of tRNA; $12,250 \mathrm{ng}$ of tRNA; 13, $1250 \mathrm{ng}$ of tRNA.

Although the minimal SLIIB probe binds Rev specifically, our data also indicate that the affinity of Rev for this probe is significantly lower than that seen with the complete SLII sequence (Fig. $3 C$ ). It therefore appears that the sequences around SLIIB could be constrained into a particularly favorable conformation by the remainder of SLII. Computer analysis (20-22) of the SLIIB probe (Fig. 3A) suggests that this RNA could fold to form a guanine-rich bulge sequence flanked on one side by the IIB stem and on the other by a short RNA helix not predicted by our earlier analysis (10) of the complete SLII RNA sequence (compare Fig. 1A). However, the formation of these two base pairs in the context of the intact RRE has been predicted by others (13). The observation that $\mathrm{A}^{75}$ is not accessible to DEPC modification under native conditions (T. Critchley and P.G.S., unpublished observations) but readily modified under denaturing conditions (Fig. 2) may also suggest that this nucleotide is base-paired in SLII (23). Recently, the formation of such a second helical region adjacent to the base of the IIB stem was proposed to be critical in Rev binding (9). Therefore the relative instability of this short RNA helix in the context of the minimal SLIIB RNA probe could contribute to the lower affinity of this RNA for Rev, when compared with the complete SLII RNA (Fig. 3C).

Several groups have reported that Rev forms multiple complexes with the RRE that can be resolved by nondenaturing PAGE (3-5, 8-10). With the SLII RRE probe, it has been shown that up to three discrete Rev-RRE complexes can be detected and that these complexes represent one, two, or three Rev protein monomers bound to a single SLII RNA molecule (9). In this report, we have attempted to identify specific nucleotides involved in the binding of more than one Rev molecule to the SLII RRE probe. Although chemical interference readily identified RRE nucleotides critically important for binding a single Rev molecule, no additional
RNA sequence requirements were observed for binding a second Rev monomer (Fig. 2).

Although this observation does not demonstrate that the binding of Rev to secondary sites is nonspecific-it could, for example, involve specific contacts with the RNA backbone that might not be detected by DEPC interference-it does suggest that such secondary binding is mechanistically distinct from the primary Rev-binding event. In this context, it is of interest to note that the M4 mutant of Rev binds to the primary Rev-binding site efficiently (Fig. 2, lane 7) but is unable to bind to any of the hypothetical secondary sites located in SLII (8). It is unlikely that M4 mutant has simply lost the ability to bind to these secondary sites, as occupation of the primary binding site by an M4 monomer also precludes occupation of the secondary sites by the wild-type Rev protein-i.e., M4 and Rev are unable to form mixed multimers on the RRE (8). Particularly relevant, we believe, is the observation that M4 mutant will not only not form multimers with the RRE but has also lost the ability to spontaneously form oligomers in the absence of RNA in concentrated solution. Daly et al. (12) noted that this correlation strongly suggests that the inability to participate in specific proteinprotein interactions underlies the inability of this Rev mutant to form multimers on the RRE.

It is of interest to compare the RNA-binding properties of Rev with those of Tat, a second essential HIV-1 regulatory protein that also binds to a structured RNA target site, the viral transactivation response element $(1,24-28)$. Tat, also an arginine-rich RNA-binding protein, can bind with high affinity to a 3-nt pyrimidine bulge in the context of two specific adjacent base pairs (24-28). Detailed biochemical analysis of this protein-RNA interaction has led to the proposal that arginine-rich RNA-binding proteins may share the property of binding specifically at the junction of single-stranded and helical RNA regions $(25,28)$, a hypothesis consistent with the binding site for Rev proposed here (Figs. $1 A$ and $3 A$ ). In 


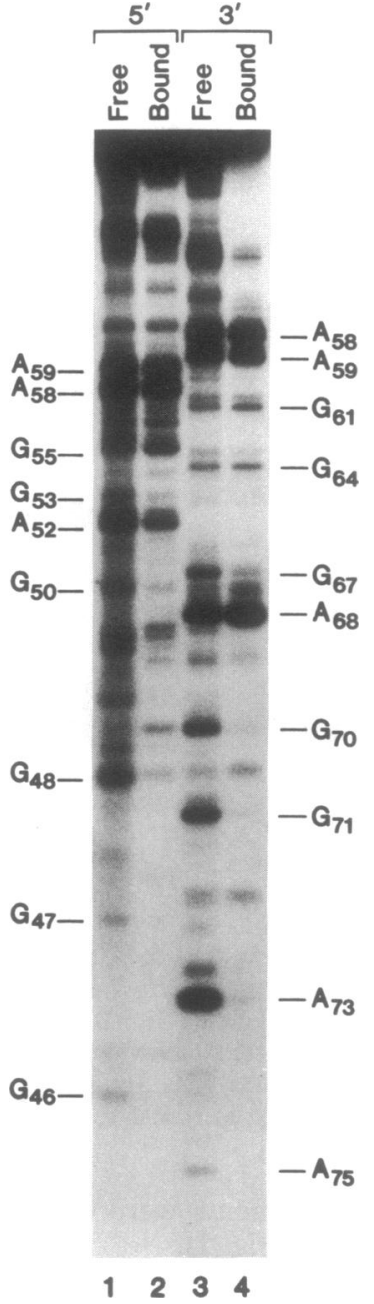

Fig. 4. Chemical interference analysis of Rev binding to the SLIIB RRE probe. A DEPC-modified SLIIB RNA probe (Fig. 3A) end-labeled at either the $5^{\prime}$ (lanes 1 and 2) or 3' (lanes 3 and 4) end was incubated with GST-Rev protein, and bound vs. free probe was separated by electrophoresis (Fig. 3B). After cleavage with aniline, $\approx 4 \times 10^{3} \mathrm{cpm}$ of each RNA sample was subjected to electrophoresis through a denaturing $20 \%$ acrylamide gel. Nucleotides that displayed chemical interference are compiled in Fig. 3A.

addition to high-affinity binding to a discrete site in the transactivation response element, Tat can also form a stable, low-affinity complex with any RNA containing a bulged helical region $(25,26)$. However, unlike the interaction with its high-affinity site, these low-affinity interactions are not detectable by modification interference $(25,26)$. It is therefore tempting to suggest that Rev, like Tat, may also interact with low-affinity sites on the basis of their specific secondary structure, independent of their primary sequence $(25)$. This hypothesis would explain our inability to detect such lowaffinity sites by modification interference-yet would also be consistent with the detection of discrete secondary binding sites for Rev on the RRE with footprinting assays (10). However, Rev appears distinct from Tat in that the binding of Rev to such secondary sites is both biologically significant and is clearly mediated, at least in part, by specific proteinprotein interactions $(8,11,12)$.

After completion of this work, Heaphy et al. (29) reported the identification of a single, high-affinity Rev-binding site within the RRE. This site, which was derived from muta- tional analysis rather than chemical interference, is essentially identical to the primary Rev-binding site mapped in this work.

We thank Sandoz Research Institute and Repligen Corp. for the gift of purified 116-amino acid Rev protein. We also thank Mariano Garcia-Blanco for helpful discussions and Sharon Goodwin for secretarial assistance. This work was supported by the Howard Hughes Medical Institute and by Public Health Service Grants AI28233, AI29821, and AI28662 from the National Institute of Allergy and Infectious Diseases to B.R.C. and by Science and Engineering Research Council Grants GR/E95750 and GR/F84409 to P.G.S.

1. Cullen, B. R. \& Greene, W. C. (1989) Cell 58, 423-426.

2. Zapp, M. L. \& Green, M. R. (1989) Nature (London) 342, 714-716.

3. Daly, T. J., Cook, K. S., Gray, G. S., Maione, T. E. \& Rusche, J. R. (1989) Nature (London) 342, 816-819.

4. Malim, M. H., Tiley, L. S., McCarn, D. F., Rusche, J. R., Hauber, J. \& Cullen, B. R. (1990) Cell 60, 675-683.

5. Heaphy, S., Dingwall, C., Ernberg, I., Gait, M. J., Green, S. M., Karn, J., Lowe, A. D., Singh, M. \& Skinner, M. A. (1990) Cell 60, 685-693.

6. Olsen, H. S., Nelbock, P., Cochrane, A. W. \& Rosen, C. A. (1990) Science 247, 845-848.

7. Malim, M. H., Hauber, J., Le, S.-Y., Maizel, J. V. \& Cullen, B. R. (1989) Nature (London) 338, 254-257.

8. Malim, M. H. \& Cullen, B. R. (1991) Cell 65, 241-248.

9. Cook, K. S., Fisk, G. J., Hauber, J., Usman, N., Daly, T. J. \& Rusche, J. R. (1991) Nucleic Acids Res. 19, 1577-1583.

10. Kjems, J., Brown, M., Chang, D. D. \& Sharp, P. A. (1991) Proc. Natl. Acad. Sci. USA 88, 683-687.

11. Olsen, H. S., Cochrane, A. W., Dillon, P. J., Nalin, C. M. \& Rosen, C. A. (1990) Genes Dev. 4, 1357-1364.

12. Daly, T. J., Cook, K. S., Fisk, G., Jensen, D., Hauber, J., Jaksche, H. \& Rusche, J. R. (1991) in Genetic Structure and Regulation of HIV, eds. Haseltine, W. A. \& Wong-Staal, F. (Raven, New York), Vol. 1, pp. 135-142.

13. Dayton, E. T., Powell, D. M. \& Dayton, A. I. (1989) Science 246, 1625-1629.

14. Holland, S. M., Ahmad, N., Maitra, R. K., Wingfield, P. \& Venkatesan, S. (1990) J. Virol. 64, 5966-5975.

15. Peattie, D. (1979) Proc. Natl. Acad. Sci. USA 76, 1760-1764.

16. Huang, X., Hope, T. J., Bond, B. L., McDonald, D., Grahl, K. \& Parslow, T. G. (1991) J. Virol. 65, 2131-2134.

17. Usman, N., Ogilvie, K. K., Jiang, M.-Y. \& Cedergren, R. J. (1987) J. Am. Chem. Soc. 109, 7845-7854.

18. Wu, T., Ogilvie, K. K. \& Pon, R. T. (1989) Nucleic Acids Res. 17, 3501-3517.

19. Talbot, S. J., Goodman, S., Bates, S. R. E., Fishwick, C. W. G. \& Stockley, P. G. (1990) Nucleic Acids Res. 18, 3521-3528.

20. Le, S.-Y., Malim, M. H., Cullen, B. R. \& Maizel, J. V. (1990) Nucleic Acids Res. 18, 1613-1623.

21. Zuker, M. \& Stiegler, P. (1981) Nucleic Acids Res. 9, 133-148.

22. Freier, S. M., Kierzek, R., Jaeger, J. A., Sugimoto, N., Caruthers, M. H., Neilson, T. \& Turner, D. H. (1986) Proc. Natl. Acad. Sci. USA 83, 9373-9377.

23. Peattie, D. A. \& Gilbert, W. (1980) Proc. Natl. Acad. Sci. USA $77,4679-4682$.

24. Roy, S., Delling, U., Chen, C.-H., Rosen, C. A. \& Sonenberg, N. (1990) Genes Dev. 4, 1365-1373.

25. Weeks, K. M. \& Crothers, D. M. (1991) Cell 66, 577-588.

26. Weeks, K. M., Ampe, C., Schultz, S. C., Steitz, T. A. \& Crothers, D. M. (1990) Science 249, 1281-1285.

27. Dingwall, C., Ernberg, I., Gait, M. J., Green, S. M., Heaphy, S., Karn, J., Lowe, A. D., Singh, M. \& Skinner, M. A. (1990) EMBO J. 9, 4145-4153.

28. Calnan, B. J., Tidor, B., Biancalana, S., Hudson, D. \& Frankel, A. D. (1991) Science 252, 1167-1171.

29. Heaphy, S., Finch, J. T., Gait, M. J., Karn, J. \& Singh, M. (1991) Proc. Natl. Acad. Sci. USA 88, 7366-7370. 